\title{
Sozialwirtschaft gestärkt
}

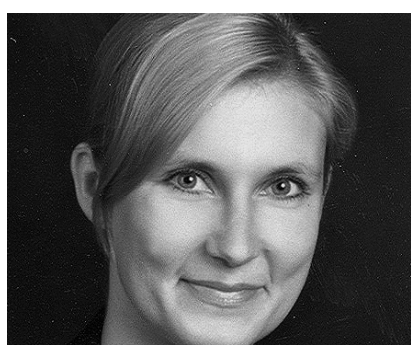

VON ULLA ENGLER

Die Rechtsanwältin Dr. Ulla Engler ist Referentin für Organisationsrecht beim Gesamtverband des Paritätischen Wohlfahrtsverbandes in Berlin.

E-Mail

organisationsrecht@paritaet.org
Dienstleistungen im sozialrechtlichen Dreiecksverhältnis sind keine öffentlichen Aufträge im Sinne des Vergaberechts. Dennoch versuchten manche Leistungsträger in der Vergangenheit, entsprechende Leistungen der Jugend-, Sozial- und Behindertenhilfe auszuschreiben. Die Reform des Vergaberechts hat nun die Stellung sozialer Unternehmen gegenüber öffentlichen Leistungsträgern gestärkt.

Vergaberecht gewinnt im sozialen Bereich zunehmend an Bedeutung. Mit der als trocken und kompliziert verschrienen Materie müssen sich Einrichtungsträger in fast allen Bereichen befassen: in der Sozialhilfe, der Kinder- und Jugendhilfe, der Arbeitsförderung und der Grundsicherung für Arbeitsuchende. Gar nicht zu sprechen von der gesetzlichen Krankenversicherung: Hier hat der Europäische Gerichtshof mit einem Urteil vom 11. Juni 2009 entschieden, dass gesetzliche Krankenkassen öffentliche Auftraggeber und damit dem Vergaberecht unterworfen sind (Az. Rs. C300/07 [AOK]).

Dieser Beitrag soll aufzeigen, wann die Vergabe sozialer Dienstleistungen zulässig ist und wann sie gegen die Vorgaben des Verfassungs- und des Sozialrechts verstößt. In einem zweiten Schritt soll umrissen werden, welche Folgen sich aus der soeben abgeschlossenen Reform des Vergaberechts für Unternehmen der Sozialwirtschaft ergeben.

\section{Anwendung von Vergaberecht auf die Erbringung sozialer Dienstleistungen}

Erteilt ein öffentlicher Auftraggeber einen öffentlichen Auftrag, so findet Vergaberecht Anwendung. Von der Höhe des Auftragswertes hängt dabei ab, ob das kartellrechtliche Vergaberecht des Gesetzes gegen Wettbewerbsbeschränkungen (GWB) oder das haushaltsrechtliche Vergaberecht der Bundeshaushaltsordnung (BHO) einschlägig ist. Bei Erreichen des Schwellenwerts von 193.000 Euro findet das kartell- rechtliche Vergaberecht Anwendung: Der Auftrag ist europaweit auszuschreiben, unterlegene Bieter können sich gegen ein fehlerhaftes Vergabeverfahren mit einem Nachprüfungsverfahren zu Wehr setzen. Unterhalb von 193.000 Euro muss der öffentliche Auftraggeber den Auftrag nur deutschlandweit ausschreiben, für unterlegene Bieter besteht so gut wie kein Rechtsschutz.

\section{Was hat die Erbringung sozialer Dienstleistungen mit Vergaberecht zu tun?}

Dass die Leistungsträger des SGB II, III, VIII und XII öffentliche Auftraggeber gem. \ 98 GWB sind, ist unbestritten. Damit müssen die Träger der Sozialhilfe, die Träger der öffentlichen Jugendhilfe und die Bundesagentur für Arbeit als Träger der Arbeitsförderung und der Grundsicherung für Arbeitsuchende Vergaberecht anwenden. Allerdings nur unter einer Voraussetzung: Sie müssen einen öffentlichen Auftrag vergeben. Und dies ist der Knackpunkt! Soziale Dienstleistungen des SGB II, VIII und XII werden in einem sozialen Dreiecksverhältnis zwischen öffentlichem Leistungsträger, Leistungserbringer und Leistungsberechtigtem erbracht (vgl. Grafik Seite 30). Ein öffentlicher Auftrag liegt nicht vor.

Hintergrund ist, dass der öffentliche Leistungsträger mit den Leistungserbringern Rahmenvereinbarungen über Art, Umfang und Qualität der Leistungen, ihre Vergütung und Abrechnung sowie über Fragen der Qualitäts-, Wirtschaftlichkeitsund Wirksamkeitsprüfung schließt. Die Leistungserbringer verpflichten sich, die vereinbarten Leistungen zu erbringen, sie erhalten als Gegenleistung aber weder eine Vergütung noch eine Belegungsgarantie. Ein Entgeltanspruch entsteht erst, wenn der Leistungsberechtigte - der aus dem Sozialrechtsverhältnis einen Rechtsanspruch auf Hilfe hat und dem der öffentliche Leistungsträger die begehrte Leistung durch Verwaltungsakt bewilligt - sich für eine bestimmte Einrichtung oder einen Dienst 


\section{Sozialrechtliches Dreiecksverhältnis:}

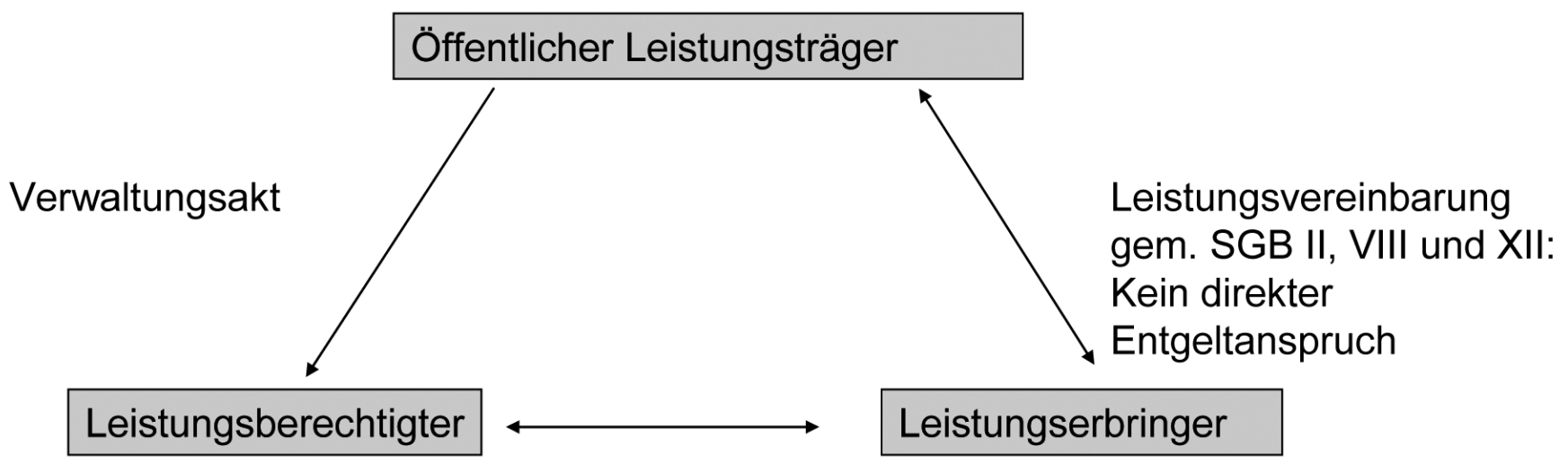

Privatrechtlicher Vertrag,

z. B. Heimvertrag als Folge der

Ausübung des Wunsch- und

Wahlrechts

Dienstleistungen im sozialen Dreiecksverhältnis zwischen öffentlichem Leistungsträger, Leistungserbringer und Leistungsberechtigtem sind keine -

öffentlichen Aufträge.

entscheidet. Erst wenn die Einrichtung eine Leistung gegenüber dem Leistungsberechtigten erbringt, erhält sie eine Vergütung.

Ganz anders verhält es sich bei einem öffentlichen Auftrag. Gemäß $\$ 99$ GWB sind öffentliche Aufträge entgeltliche Verträge von öffentlichen Auftraggebern mit Unternehmen über die Beschaffung von Leistungen, die Liefer-, Bau- oder Dienstleistungen zum Gegenstand haben. Es muss folglich ein Gegenseitigkeitsverhältnis vorliegen, in dem das Unternehmen eine Leistung erbringt und hierfür eine Gegenleistung - nämlich die Vergütung erhält. In einem dreiseitigen Sozialrechtsverhältnis hingegen erbringt zwar der Leistungserbringer eine Leistung an den Leistungsberechtigten, er erhält jedoch von ihm keine Vergütung. Eine Vergütung erhält er zwar vom Sozialleistungsträger, an diesen erbringt er jedoch keine Leistung. Es liegt also keine rechtliche Verknüpfung von Leistung und Gegenleistung im sozialrechtlichen Dreiecksverhältnis vor.

Damit steht fest, dass mangels eines öffentlichen Auftrags Vergaberecht keine Anwendung findet, wenn die Leistungserbringung im Rahmen eines sozialrechtlichen Dreiecksverhältnisses erfolgt. Dies ist im SGB II, VIII und XII der Fall, nicht je- doch im SGB III. Überwiegend wird angenommen, dass die Vereinbarungen zur Erbringung sozialer Dienstleistungen vielmehr Dienstleistungskonzessionen ähneln. Auch Dienstleistungskonzessionen sind vom Anwendungsbereich des Vergaberechts ausgenommen.

Schreiben öffentliche Leistungsträger Leistungen des SGB II, VIII und XII dennoch aus, so verstoßen sie sowohl gegen Sozialrecht als auch gegen Verfassungsrecht. So haben zahlreiche Verwaltungsund Oberverwaltungsgerichte entschieden, dass nicht nur das Grundrecht der Leistungsberechtigten aus Art. 2 Abs. 1 GG sowie ihre Wunsch- und Wahlrecht aus den Sozialgesetzbüchern verletzt werden, wenn ihnen keine Wahl der Leistungserbringers, ggf. nach ihrer Konfession, möglich ist. Auch wird in die Rechte der Einrichtungen und Dienste eingegriffen, die im Vergabeverfahren nicht den $\mathrm{Zu}-$ schlag erhalten und somit keine Leistungen mehr gegenüber den Leistungsberechtigten mehr erbringen dürfen. Sie werden nicht nur in ihrem Grundrecht auf Berufsfreiheit aus Art. 12 Abs. 1 GG, sondern auch in ihrem Anspruch auf ermessensfehlerfreie Entscheidung durch den öffentlichen Leistungsträger verletzt (vgl. Kasten mit Buchhinweis auf Seite 31).

\section{Reform des Vergaberechts}

In der letzten Legislaturperiode wurde die lang angekündigte Reform des Vergaberechts in Angriff genommen. Die Reform bestand zum einen aus einer Änderung des Gesetzes gegen Wettbewerbsbeschränkungen, zum anderen aus einer Neufassung der Vergabe- und Vertragsordnung für Bauleistungen (VOB) und der Vergabe- und Vertragsordnung für Leistungen - Teil A (VOL/A).

\section{Reform des Gesetzes gegen Wettbewerbsbeschränkungen}

Die Änderung des Gesetzes gegen Wettbewerbsbeschränkungen trat zum 24. April 2009 mit dem »Gesetz zur Modernisierung des Vergaberechts « in Kraft. Ziel waren sowohl die längst fällige Umsetzung der europäischen Vergaberichtlinien 2004/18/EG und 2004/17/EG in nationales Recht sowie sind die Vereinfachung, Modernisierung und Flexibilisierung des deutschen Vergaberechts. Für Unternehmen der Sozialwirtschaft sind insbesondere folgende Änderungen von Bedeutung:

- Mittelstandsschutz: Unter dem Stichwort » Mittelstandsschutz « soll die mit- 
telständische Wirtschaft - und damit auch kleinere Einrichtungen und Dienste-gem. $\$ 97$ Abs. 3 GWB bei der Auftragsvergabe stärker als in der Vergangenheit berücksichtigt werden. Dies soll u. a. dadurch geschehen, dass bei der Vergabe eines öffentlichen Auftrags die Leistungen in der Menge aufgeteilt (Teillose) und getrennt nach Fachgebiet (Fachlose) zu vergeben sind. Nur in begründeten Ausnahmefällen kann davon abgewichen werden, wenn wirtschaftliche oder technische Gründe dies erfordern.

- Berücksichtigung sozialer Kriterien bei der Auftragsvergabe: Bei der Auftragsvergabe können nunmehr gem. $\mathbb{S} 97$ Abs. 4 GWB zusätzliche Kriterien wie soziale, umweltbezogene und innovative Aspekte Berücksichtigung finden. Allerdings handelt es sich bei der Vorschrift um eine Kann-Formulierung, die dem Auftraggeber die Wahlmöglichkeit lässt, ob er die zusätzlichen Anforderungen bei der Auftragsvergabe von Bietern berücksichtigt wissen möchte. Zwar konnte auch im Rahmen der alten Rechtslage der öffentliche Auftraggeber neben den im Gesetz ausdrücklich normierten Anforderungen des Fachwissens, der Leistungsfähigkeit, der Zuverlässigkeit und der Wirtschaftlichkeit weitere Aspekte berücksichtigen. Voraussetzung war jedoch, dass dies durch Bundes- oder Landesgesetz ausdrücklich geregelt war.

- Bestimmte soziale Dienstleistungen sind vom Vergaberecht ausgenommen: \99 Abs. 1 Satz 1 GWB trifft erstmalig eine ausdrückliche Festlegung, dass es sich bei Baukonzessionen um öffentliche Aufträge handelt. Im Umkehr- schluss kann es sich - auch nach der Gesetzesbegründung - bei Dienstleistungskonzessionen daher nicht um öffentliche Aufträge handeln. Dieser Umstand ist insbesondere für gemeinnützige Träger von Bedeutung, die soziale Dienstleistungen im Bereich der Grundsicherung für Arbeitsuchende (SGB II), der Jugendhilfe (SGB VIII) und der Sozialhilfe (SGB XII) erbringen. Diese Leistungen werden nicht im
Wettbewerbsbeschränkungen ausgenommen sind. Dennoch stellt auch die Neuregelung einen Fortschritt dar.

\section{Novellierung von VOB und VOL}

Am 11. Juni 2010 traten die neue Vergabe- und Vertragsordnung für Bauleistungen (VOB) sowie die neue Vergabeund Vertragsordnung für Leistungen - Teil A (VOL/A) in Kraft. Zwar wurden bereits

\section{"Öffentliche Leistungsträger verstoßen gegen Sozialrecht und Verfassung, wenn sie Leistungen nach SGB II, VIII und XII \\ ausschreiben"}

\begin{abstract}
Rahmen eines öffentlichen Auftrags, sondern im Rahmen eines sozialrechtlichen Dreiecksverhältnisses erbracht. Zwar ist nicht gesetzlich geregelt, dass Leistungen im Rahmen eines sozialrechtlichen Dreiecksverhältnisses eine Dienstleistungskonzession darstellen, überwiegend wird jedoch angenommen, dass es sich um ein Vertragsverhältnis »ähnlich « einer Dienstleistungskonzession handelt. Zwar wäre, um Rechtsunsicherheiten zu vermeiden, eine Klarstellung im Gesetz gegen Wettbewerbsbeschränkungen wünschenswert gewesen, dass im sozialrechtlichen Dreiecksverhältnis zu erbringende Dienstleistungen vom Anwendungsbereich des Vierten Teils des Gesetz gegen
\end{abstract}

am 15. Oktober 2009 die überarbeitete VOB im Bundesanzeiger Nr. 155a und am 29. Dezember 2009 die überarbeitete VOL/A im Bundesanzeiger Nr. 196a bekanntgemacht. Jedoch bedurfte es noch des Inkrafttretens der Vergabeverordnung zum 11. Juni 2010, um beide Regelwerke rechtswirksam werden zu lassen.

Ursprünglich war geplant, die Vergabeund Vertragsordnung für Bauleistungen um eine Regelung zu ergänzen, nach der "gemeinnützige Unternehmen « vom Wettbewerb mit gewerblichen Unternehmen bei der Vergabe von Bauleistungen ausgenommen werden. Behindertenwerkstätten und Integrationsbetriebe wären damit aus Vergabeverfahren für Bauleistungen ausgeschlossen worden.

\section{Das Vergaberecht in der Sozialwirtschaft}

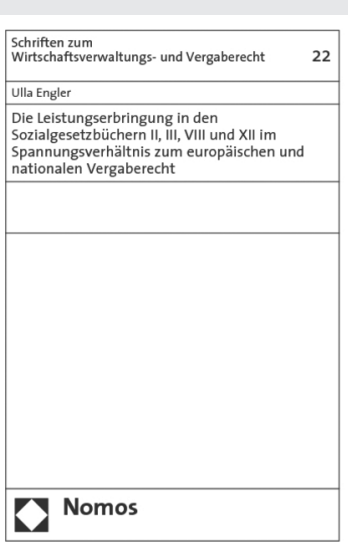

In Zeiten knapper öffentlicher Kassen ist das Vergaberecht auch im sozialen Bereich zunehmend von Bedeutung. Hierbei stellt sich jedoch die Frage nach der Vereinbarkeit von Sozialrecht und Vergaberecht. Vor diesem Hintergrund stellt die Verfasserin anhand aktueller Rechtsprechung die Leistungserbringung in den Bereichen der Grundsicherung für Arbeitsuchende, der Arbeitsförderung, der Kinder- und Jugendhilfe und der Sozialhilfe dar und zeigt auf, wo die sozialrechtliche Leistungserbringung im Spannungsverhältnis zum Vergaberecht steht. Während das europäische Vergaberecht in erster Linie Rahmenbedingungen vorgibt, resultieren aus dem nationalen Vergaberecht konkrete Vorgaben, die öffentliche Auftraggeber bei der Vergabe öffentlicher Aufträge zu beachten haben. Schwerpunkt der Arbeit ist die Frage, ob bei der Leistungserbringung in den SGB II, III, VIII und XII, die vorwiegend im Rahmen eines sozialrechtlichen Dreiecksverhältnisses erbracht wird, überhaupt ein öffentlicher Auftrag im Sinne des Vergaberechts vorliegt. Die Autorin verfügt als Rechtsanwältin bei einem Paritätischen Wohlfahrtsverband als einem Spitzenverband der Freien Wohlfahrtspflege über praktische Erfahrung aus beiden Welten, dem Sozialrecht und dem Vergaberecht.

Ulla Engler: Die Leistungserbringung in den Sozialgesetzbüchern II, III, VIII und XII im Spannungsverhältnis zum europäischen und nationalen Vergaberecht. Nomos Verlagsgesellschaft, Baden-Baden 2010. 221 Seiten. 59,- Euro. ISBN 978-3-8329-5315-7. 
Auch aufgrund des umfangreichen Engagements des Paritätischen Wohlfahrtsverbandes gegenüber dem zuständigen Bundesministerium, dem Bundestagsausschuss für Verkehr, Bau und Stadtentwicklung sowie einzelnen Abgeordneten wurde der geplante Ausschluss gemeinnütziger Unternehmen in der Vergabe- und Vertragsordnung für Bauleistungen jedoch nicht verwirklicht. Zwar ist in $\mathbb{} 6$ Abs. 1 Nr. 3 VOB/A weiterhin formuliert, dass »Justizvollzugsanstalten, Einrichtungen der Jugendhilfe, Aus- und Fortbildungsstätten und ähnliche Einrichtungen sowie Betriebe der öffentlichen Hand und Verwaltungen « zum Wettbewerb mit gewerblichen Unternehmen nicht zuzulassen sind. Aufgrund der Rechtsprechung des OLG Düsseldorf zu dieser Formulierung steht jedoch unstrittig fest, dass gemeinnützige Organisationen nicht unter diesen Ausschluss zu subsumieren sind.

Für Erbringer sozialer Dienstleistungen ist in diesem Zusammenhang auch die Änderung der VOL/A - insbesondere im Bereich der Arbeitsmarktdienstleistungen von Bedeutung. In der VOL/A sollte im Gegensatz zur Vergabe- und Vertragsordnung für Bauleistungen von vornherein kein Ausschluss gemeinnütziger Unternehmen vom Wettbewerb stattfinden. Vielmehr wurde - wie von Wohlfahrtsverbänden mehrfach gefordert $-\$ 7 \mathrm{Nr}$. 6 VOL/A (der dem Wortlaut des $\$ 6$ Abs. 1 Nr. 3 $\mathrm{VOB} / \mathrm{A}$ weitgehend entsprach) bis auf Justizvollzugsanstalten komplett gestrichen. Damit ist eine Klarstellung der früher so umstrittenen Ausschlussregelung erfolgt.

\section{Welfare Mix in der Altenhilfe}

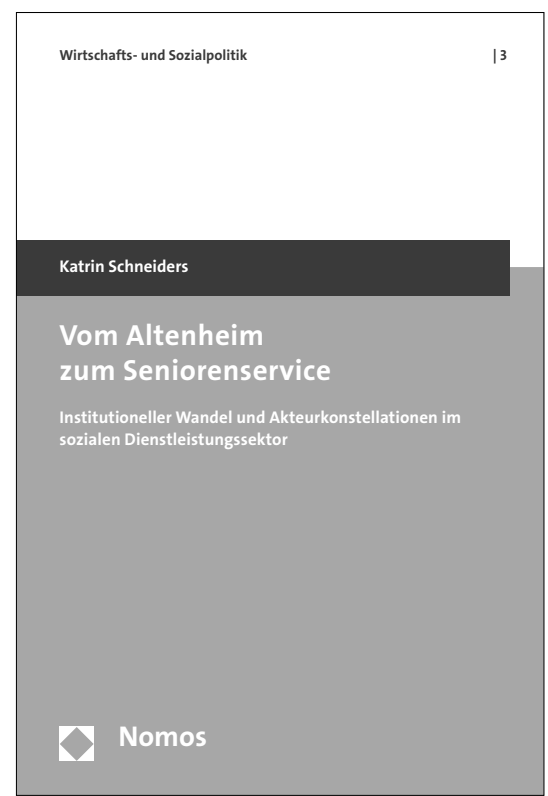

\section{Vom Altenheim zum Seniorenservice}

Institutioneller Wandel und Akteurkonstellationen im sozialen Dienstleistungssektor

Von Dr. Katrin Schneiders

2010, 268 S., brosch., 54,-€, ISBN 978-3-8329-5347-8

(Wirtschafts- und Sozialpolitik, Bd. 3)
Der soziale Dienstleistungssektor ist seit den 199oer Jahren einem grundlegenden Wandel unterworfen, der mit den Stichworten Ökonomisierung, Professionalisierung und Entgrenzung erfasst werden kann. Hiervon sind insbesondere die Wohlfahrtsverbände betroffen.

Bislang haben die frei-gemeinnützigen Anbieter im Pflegesektor ihre dominierende Position weitgehend verteidigen können. Gleichwohl sind Verschiebungen zwischen den einzelnen Trägertypen erkennbar. Dies ist das Ergebnis einer empirischen Analyse von Informationen aus ca. 10.000 Einrichtungen der Altenpflege, die im Buch vorgestellt wird. Darüber hinaus werden vor dem Hintergrund pluralisierter Lebensformen und Bedürfnisse älterer Menschen differenzierte Daten zur Nachfrageentwicklung sowie zu Umfang und Struktur neuer Wohnformen präsentiert.

Angesichts des fortschreitenden demographischen Wandels, der erheblichen beschäftigungspolitischen Bedeutung des Sektors sowie der beschränkten Marktfähigkeit der Pflege sind neue Steuerungsformen jenseits von Markt und Staat erforderlich. Auf der Suche nach Lösungsansätzen wird diskutiert, welche Rolle die Wohlfahrtsverbände im zukünftigen Welfare Mix übernehmen können.

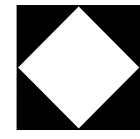

\section{Nomos}

